\title{
Effect of soy isoflavones on breast cancer recurrence and death for patients receiving adjuvant endocrine therapy
}

\author{
Xinmei Kang MD PhD, Qingyuan Zhang MD PhD, Shuhuai Wang MD, Xu Huang MD, Shi Jin MD
}

Previously published at www.cmaj.ca

\section{ABSTRACT}

Background: The intake of soy isoflavones among women with breast cancer has become a public health concern, because these compounds have weak estrogenic effects. There is little clinical evidence about their safety for patients with breast cancer who are receiving adjuvant endocrine therapy.

Methods: For patients who underwent surgery for breast cancer between August 2002 and July 2003 and who were receiving adjuvant endocrine therapy, we examined associations between dietary intake of soy isoflavones and recurrence of breast cancer and death. We measured dietary intake of soy isoflavones at baseline using a validated food frequency questionnaire. We estimated hazard ratios (HRs) and $95 \%$ confidence intervals (Cls) by means of multivariable Cox proportional hazards regression models. We further stratified the analyses by hormonal receptor status and endocrine therapy.

Results: The median follow-up period for the 524 patients in this study was 5.1 years. Among premenopausal patients, the overall death rate $(30.6 \%)$ was not related to intake of soy isoflavones ( $\mathrm{HR}=1.05,95 \% \mathrm{Cl} 0.78-1.71$ for the highest quartile [ $>42.3 \mathrm{mg} /$ day] $\mathrm{v}$. the lowest quartile $[<15.2 \mathrm{mg} /$ day $], p$ for trend $=0.87$ ). Relative to postmenopausal patients in the lowest quartile of soy isoflavone intake, the risk of recurrence for postmenopausal patients in the highest quartile was significantly lower $(\mathrm{HR}=0.67,95 \% \mathrm{Cl} 0.54-0.85, p$ for trend $=$ 0.02 ). Inverse associations were observed in patients with estrogen and progesterone receptor positive disease and those receiving anastrozole therapy.

Interpretation: High dietary intake of soy isoflavones was associated with lower risk of recurrence among postmenopausal patients with breast cancer positive for estrogen and progesterone receptor and those who were receiving anastrozole as endocrine therapy.

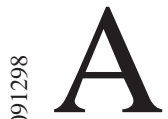

variety of health benefits in terms of cancer and cardiovascular disease have been attributed to the consumption of soy foods, primarily because of soy isoflavones. ${ }^{1}$ Three primary isoflavones account for virtually all of the isoflavones in soy beans: genistein (about 50\%), daidzein (about 40\%) and glycitein (about 10\%). The chemical structure of soy isoflavones is similar to that of estrogens. The isoflavones are therefore considered to be possible select- ive estrogen receptor modulators, which may bind to estrogen receptors and selectively stimulate or inhibit estrogen-like action in various tissues. ${ }^{2}$ Given that soy-based foods are now more frequently consumed than was previously the case, both as an alternative approach to treating the symptoms of menopause and for promoting cardiovascular health, ${ }^{3,4}$ concerns have arisen about the intake of these compounds by patients with hormone-sensitive breast cancer, in whom tumour growth depends largely on estrogen. Tamoxifen and anastrozole are commonly used as adjuvant endocrine therapy for hormone-sensitive breast cancer, and these drugs are effective in preventing recurrence and prolonging survival. ${ }^{5,6}$ In some experimental studies the inhibitory effects of tamoxifen on growth of implanted mammary tumours were negated by dietary administration of soy isoflavones, ${ }^{7.8}$ but in other rodent cancer models, soy food appeared to enhance the beneficial effects of tamoxifen. ${ }^{9}$ Little is known about the potential effects of consuming soy isoflavones for patients with breast cancer who are receiving adjuvant endocrine therapy. We used data for a cohort of postoperative patients with breast cancer who were receiving adjuvant endocrine therapy to examine the relation between intake of soy isoflavones and recurrence of breast cancer and death.

\section{Methods}

\section{Study participants}

From August 2002 to July 2003, we recruited women with a diagnosis of early or local advanced breast cancer who were receiving adjuvant endocrine therapy after surgery at the Cancer Hospital of Harbin Medical University in Harbin, China. We identified potentially eligible patients by reviewing the hospital's pathological diagnosis records after surgery for breast cancer. The institutional review board of Harbin Medical University provided ethics approval. For each patient, we contacted the attending physician to confirm eligibility, and asked the physician to contact the patient on our behalf. Two pathologists independently confirmed the hormonal receptor status of the tumours by reviewing the results of streptavidin peroxidase immunohistochemical staining. The cut-off for positivity was

From the Departments of Medical Oncology (Kang, Zhang, Huang, Jin) and Pathology (Wang), Cancer Hospital of Harbin Medical University, Harbin, China

CMAJ 2010. DOI:10.1503/cmaj.091298 
set at $10 \%$ nuclear staining of tumour cells for estrogen receptor or progesterone receptor. The criteria for menopause were age 60 years or older; age less than 60 years and amenorrheic for at least 12 months; or age less than 60 years and amenorrheic for less than 12 months, with follicle-stimulating hormone and estradiol in the postmenopausal range. Patients were treated with tamoxifen $20 \mathrm{mg} /$ day or anastrozole $1 \mathrm{mg}$ /day as adjuvant endocrine therapy for five years, unless adverse effects were unacceptable or the disease progressed.

\section{Assessment of dietary intake of soy isoflavones}

After obtaining informed consent, we asked each patient to complete a comprehensive questionnaire covering demographic characteristics, physical activity and usual diet. The food frequency questionnaire was modelled after a validated instrument ${ }^{10}$ and included questions about usual consumption of soymilk, soy flour, dry soybeans, fresh soybeans, soybean sprouts, tofu and other soy products. We also conducted an interview, during which we asked each participant about the frequency of consumption of various soy foods (daily, weekly, monthly or yearly) and the amount eaten per unit of time (day, week, month or year) in the previous five years. We then calculated the intake of soy isoflavones using the food composition database published by the Chinese Center for Disease Control and Prevention, which provides the isoflavone content of selected foods. ${ }^{11}$ We categorized the mean daily intake of soy isoflavones in quartiles. We also asked all participants whether their intake of soy foods after diagnosis was similar to, greater than or less than their usual intake of these foods in the previous five years.

Table 1: Demographic and clinical characteristics of 524 patients with breast cancer

\begin{tabular}{|c|c|c|c|c|c|}
\hline \multirow[b]{2}{*}{ Characteristic } & \multicolumn{5}{|c|}{ Event; no. (\%) of patients in category } \\
\hline & $\begin{array}{c}\text { Total } \\
n=524\end{array}$ & $\begin{array}{c}\text { Recurrence } \\
n=185\end{array}$ & $p$ value & $\begin{array}{l}\text { Death } \\
n=154\end{array}$ & $p$ value \\
\hline Age at diagnosis, yr & & & 0.030 & & 0.73 \\
\hline$<45$ & $104(100)$ & $43(41.3)$ & & $32(30.8)$ & \\
\hline $45-54$ & $197(100)$ & $76(38.6)$ & & $62(31.5)$ & \\
\hline $55-64$ & $156(100)$ & $52(33.3)$ & & $43(27.6)$ & \\
\hline$>64$ & $67(100)$ & $14(20.9)$ & & $17(25.4)$ & \\
\hline Menopausal status & & & 0.24 & & 0.55 \\
\hline Premenopausal & $248(100)$ & $94(37.9)$ & & $76(30.6)$ & \\
\hline Postmenopausal & $276(100)$ & $91(33.0)$ & & $78(28.3)$ & \\
\hline TNM stage & & & $<0.001$ & & $<0.001$ \\
\hline I & $64(100)$ & $10(15.6)$ & & $8(12.5)$ & \\
\hline II & $345(100)$ & $96(27.8)$ & & $81(23.5)$ & \\
\hline III & $115(100)$ & $79(68.7)$ & & $65(56.5)$ & \\
\hline ER/PR status & & & 0.88 & & 0.28 \\
\hline $\mathrm{ER}+/ \mathrm{PR}+$ & $295(100)$ & $102(34.6)$ & & $79(26.8)$ & \\
\hline $\mathrm{ER}+/ \mathrm{PR}-$ & $152(100)$ & $54(35.5)$ & & $48(31.6)$ & \\
\hline ER-/PR+ & $77(100)$ & $29(37.7)$ & & $27(35.1)$ & \\
\hline Endocrine therapy & & & 0.41 & & 0.94 \\
\hline Tamoxifen & $438(100)$ & $158(36.1)$ & & $129(29.5)$ & \\
\hline Anastrozole & $86(100)$ & $27(31.4)$ & & $25(29.1)$ & \\
\hline Chemotherapy & & & 0.055 & & 0.014 \\
\hline Yes & $446(100)$ & $150(33.6)$ & & $122(27.4)$ & \\
\hline No & $78(100)$ & $35(44.9)$ & & $32(41.0)$ & \\
\hline Radiotherapy & & & 0.056 & & 0.57 \\
\hline Yes & $55(100)$ & $13(23.6)$ & & $18(32.7)$ & \\
\hline No & $469(100)$ & $172(36.7)$ & & $136(29.0)$ & \\
\hline Soy isoflavones & & & 0.41 & & 0.97 \\
\hline$<15.2$ & $132(100)$ & $54(40.9)$ & & $39(29.5)$ & \\
\hline $15.3-25.4$ & $132(100)$ & $47(35.6)$ & & $37(28.0)$ & \\
\hline $25.5-42.3$ & $130(100)$ & $43(33.1)$ & & $40(30.8)$ & \\
\hline$>42.3$ & $130(100)$ & $41(31.5)$ & & $38(29.2)$ & \\
\hline
\end{tabular}

Note: $\mathrm{ER}=$ estrogen receptor, $\mathrm{PR}=$ progesterone receptor. 


\section{Follow-up}

We followed patients until July 2008. From the medical records of patients who could be contacted at that time, we obtained complete clinical data for our analyses, including age at diagnosis, medical history, Karnofsky performance status, menopause status, pathological diagnosis, clinical stage of cancer, treatment (surgery, chemotherapy, radiotherapy and endocrine therapy), response to treatment and overall outcome. We also obtained information about disease progress, date of recurrence (if any), and date and cause of death (if deceased). For participants who could not be contacted, we established survival status by linkage to a mortality registry.

\section{Statistical analysis}

We used Cox proportional hazards regression to estimate hazard ratios (HRs) and 95\% confidence intervals (CIs) for the association between intake of soy isoflavones and recurrence or death, with HR values above 1.0 indicating increased risk. We calculated the sample size for Cox regression using the method reported by Hsieh and Lavori. ${ }^{12}$ According to our preliminary results, we needed a sample of 246 observations to achieve $80 \%$ power at a significance level of $p=0.05$ to detect an anticipated death rate of $30 \%$. In the multivariable analyses for premenopausal and postmenopausal patients, we adjusted the models for the following known risk factors related to breast cancer survival: age at diagnosis, TNM stage, hormonal receptor status, chemotherapy, radiotherapy and endocrine therapy. We developed our final multivariable models using stepwise selection, allowing variables to remain in the model if they were significant at the 0.05 level. We applied the restricted cubic spline function in Cox regression analyses to test for the proportional hazards assumption and to evaluate the pattern of association between intake of soy isoflavones and recurrence or death. ${ }^{13}$ To test for linear trends across quartiles of soy isoflavone intake, we assigned the median value for the study population to each category and used these values as a continuous variable. We conducted analyses stratified by estrogen and progesterone receptor status and by endocrine therapy to evaluate whether these hormonal factors modified the association between intake of soy isoflavones and recurrence of breast cancer. All statistical tests were based on two-tailed probability and a significance level set at $\alpha<0.05$.

\section{Results}

Of 565 eligible patients whose physicians agreed they could participate, 524 enrolled and completed the questionnaire. At the time of diagnosis, the patients ranged in age from 29 to 72 years (Table 1). Of the 524 patients, $248(47.3 \%)$ were premenopausal, $276(52.7 \%)$ were postmenopausal, $446(85.1 \%)$ received adjuvant chemotherapy, and $55(10.5 \%)$ underwent postoperative radiotherapy. All 248 of the premenopausal patients and 190 of the postmenopausal patients received tamoxifen as adjuvant endocrine therapy, and 86 of the postmenopausal patients received anastrozole. All of the patients had cancer that was positive for estrogen receptor, positive for progesterone receptor or positive for both (Table 1). The median interval between surgery and the study interview was 120 days.

In these patients, soymilk accounted for $26 \%$ of total intake of soy isoflavones, followed by tofu (21\%) and soy flour $(15 \%)$. The mean daily intake of isoflavones was $25.6 \mathrm{mg} / \mathrm{day}$ for all patients. Within the quartiles for isoflavone consumption, the mean intake was $6.5,18.2,35.8$ and $49.6 \mathrm{mg} /$ day. These amounts fall within the average range of intake of soy isoflavones in Asian countries (25-50 mg/day). ${ }^{14}$ All participants completed the supplementary survey about intake of soy foods. Most participants (452 [86.3\%]) reported no change in intake of soy foods over the previous five years, 29 (5.5\%) reported an increase and $43(8.2 \%)$ reported a decrease.

Of the original 524 patients, we were able to contact 508 directly for follow-up in July 2008. Among the 16 patients who could not be contacted directly, the mortality registry revealed 10 deaths. For six patients there was no match in the registry, and we assumed that they were still living. The total number of deaths was 154 , and 132 of these were due to breast cancer. The other causes of death were cardiovascular disease (12 patients), respiratory disease (5), other cancers (2) and other causes (3).

The five-year recurrence rate of premenopausal patients was higher than that of postmenopausal patients, but this dif-

Table 2: Adjusted association between soy isoflavones and recurrence of breast cancer or death in 248 premenopausal and 276 postmenopausal patients (total $n=524$ )

\begin{tabular}{|c|c|c|c|c|c|c|c|c|}
\hline $\begin{array}{l}\text { Soy } \\
\text { isoflavones, } \\
\mathrm{mg} / \mathrm{d}\end{array}$ & \multicolumn{4}{|c|}{ Premenopausal } & \multicolumn{4}{|c|}{ Postmenopausal } \\
\hline$<15.2$ & $29 / 68$ & 1.00 & $21 / 68$ & 1.00 & $25 / 64$ & 1.00 & $18 / 64$ & 1.00 \\
\hline$>42.3$ & $21 / 59$ & $0.88(0.61-1.23)$ & $19 / 59$ & $1.05(0.78-1.71)$ & $20 / 71$ & $0.67(0.54-0.85)$ & $19 / 71$ & $0.88(0.56-1.24)$ \\
\hline$p$ for trend & & 0.46 & & 0.87 & & 0.02 & & 0.76 \\
\hline
\end{tabular}

Note: $\mathrm{Cl}=$ confidence interval, $\mathrm{HR}=$ hazard ratio.

*Adjusted for age at diagnosis, TNM stage, estrogen and progesterone receptor status, chemotherapy and radiotherapy. Values of HR $>1$ indicate increased risk. 
ference was not statistically significant. There was a trend for an increase in rates of recurrence and death with worsening TNM stage $(p<0.01)$. The rates of recurrence and death did not vary significantly with estrogen and progesterone receptor status or with endocrine therapy.

\section{Menopausal status}

There was no association between intake of soy isoflavones and recurrence or death among premenopausal patients, and this relationship did not vary by selected prognostic factors, including age at diagnosis, hormonal receptor status, TNM stage or cancer treatment (Table 2). Among the postmenopausal patients, an inverse association was observed between intake of soy isoflavones and recurrence. Compared with postmenopausal patients in the lowest quartile of soy isoflavone intake $(<15.2 \mathrm{mg} / \mathrm{day})$, those in the highest quartile (> $42.3 \mathrm{mg} /$ day) had a significantly lower risk of recurrence (HR $0.67,95 \%$ CI $0.54-0.85, p$ for trend $=0.02)$. In contrast, there was no association between soy isoflavone intake and death in postmenopausal patients. The results of analyses for mortality specific to breast cancer were similar to those for all-cause mortality (HR 0.92, 95\% CI 0.60-1.31, $p$ for trend $=0.82$ for risk of death). We also examined the association between intake of soy isoflavones and recurrence or death among patients with no change in intake over time, to determine whether a change in intake after diagnosis might alter these associations. The associations were similar for those patients (HR 0.68, 95\% CI 0.59-0.87, $p$ for trend $=0.02$ for risk of recurrence among postmenopausal patients).

\section{Hormonal receptor status and endocrine therapy}

We stratified the analyses for risk of recurrence according to estrogen and progesterone receptor status and endocrine therapy for postmenopausal patients. Statistically significant inverse associations between intake of soy isoflavones and recurrence were observed for patients with estrogren- and progesteronepositive disease and for patients receiving anastrozole therapy (Figure 1). Among postmenopausal patients with estrogren- and progesterone-positive breast cancer, those in the highest quartile of isoflavone intake had a significantly lower risk of recurrence than those in the lowest intake quartile (HR 0.66, 95\% CI 0.49 $0.86, p$ for trend $=0.01)$. The recurrence rate of estrogren- and progesterone-positive breast cancer was $12.9 \%$ lower among patients in the highest quartile of soy isoflavone intake than among those in the lowest quartile. Among postmenopausal patients receiving anastrozole therapy, those in the highest quartile of isoflavone intake had a significantly lower risk of recurrence than those in the lowest intake quartile (HR 0.65, 95\% CI

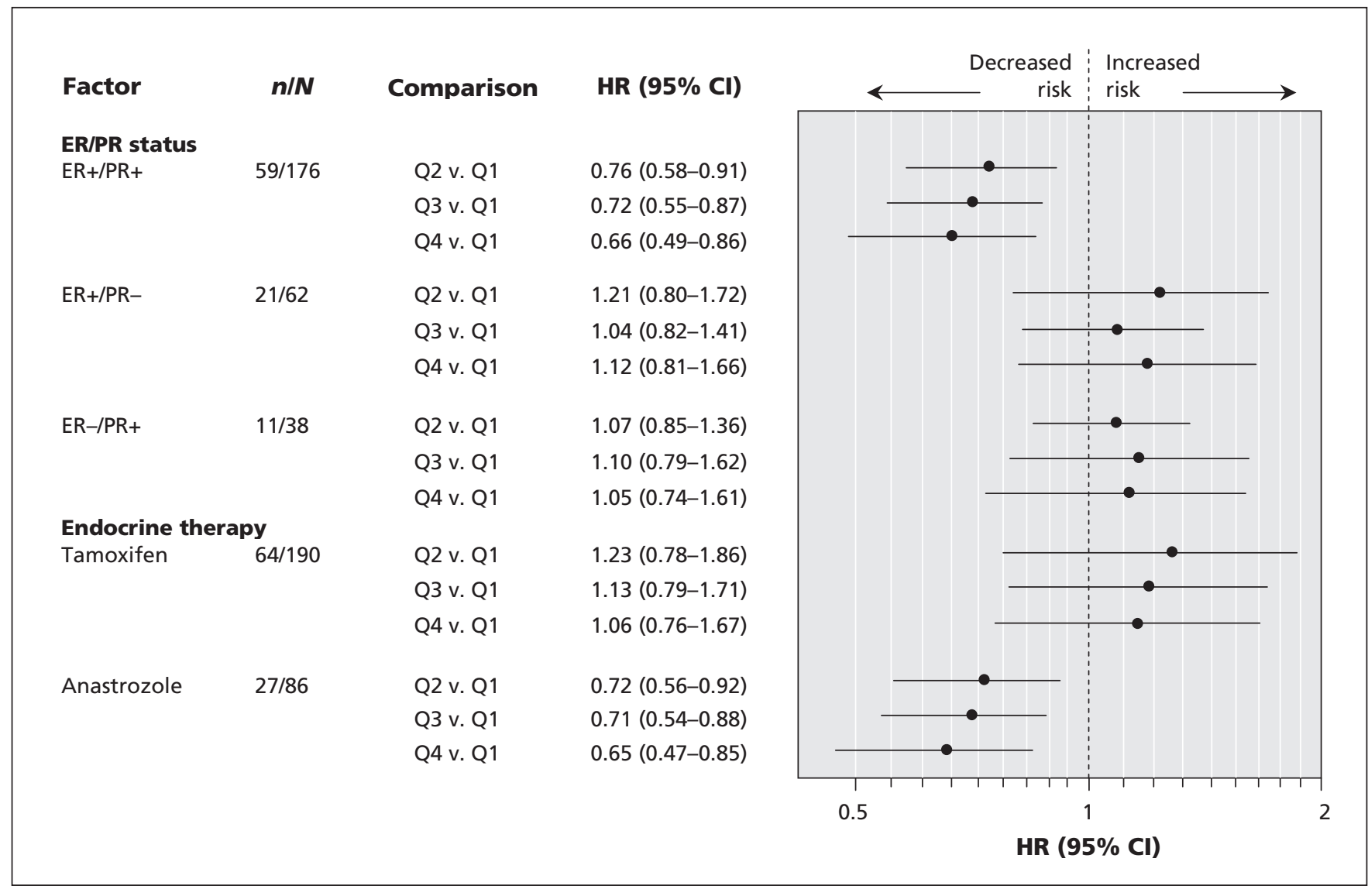

Figure 1: Forest plot of adjusted hazard ratios (HRs) for the effect of intake of soy isoflavones on recurrence among postmenopausal patients with breast cancer, stratified by estrogen and progesterone receptor status and endocrine therapy. $\mathrm{Cl}=\mathrm{confidence}$ interval, $\mathrm{ER}=$ estrogen receptor, $\mathrm{PR}=$ progesterone receptor, $\mathrm{Q} 1$ to Q4 = quartiles according to intake of soy isoflavones, where Q1 is $<15.2$ $\mathrm{mg} /$ day and Q4 is $>42.3 \mathrm{mg} /$ day. 
$0.47-0.85, p$ for trend $=0.005)$. The recurrence rate for postmenopausal patients receiving anastrozole therapy was $18.7 \%$ lower among patients in the highest quartile of soy isoflavone intake than those in the lowest quartile.

\section{Interpretation}

In this study, intake of soy isoflavones did not affect recurrence and death among premenopausal patients with breast cancer who were receiving endocrine therapy. However, postmenopausal patients in the highest quartile of soy isoflavone intake had a lower risk of recurrence while they were receiving endocrine therapy. There was no statistically significant difference in physical activity or other aspects of usual diet between patients eating high and low amounts of soy food, which would appear to exclude any effect of these lifestyle factors in the risk analyses.

Epidemiologic evidence has suggested that exposure to relatively high concentrations of soy isoflavones during adolescence may have a protective effect in relation to breast cancer during adulthood. ${ }^{15,16}$ However, the consumption of soy isoflavone supplements (as hormone replacement therapy) by women at high risk of breast cancer and by breast cancer survivors is still controversial. Concern has arisen that soy isoflavones may have a detrimental effect in these women because they may stimulate the growth of estrogen-sensitive breast tumours. Until now, clinical research has been limited, and there is little clinical evidence to suggest that intake of soy isoflavones increases the risk of breast cancer among healthy women or worsens the prognosis for those who have breast cancer. ${ }^{17}$ The results of the Shanghai Breast Cancer Study, conducted between 1996 and 1998, showed that soy intake was unrelated to disease-free breast cancer survival, but the authors of that study did not take endocrine therapy into consideration in their survival analyses. ${ }^{18} \mathrm{~A}$ recent epidemiologic study involving 1954 female survivors of breast cancer also suggested a lower risk of breast cancer recurrence with high intake of daidzein and glycetin among both postmenopausal women and tamoxifen users. ${ }^{19}$

After menopause, despite the loss of ovarian hormones, most estrogens are formed in the peripheral tissues and exert their effects locally in a paracrine or intracrine manner. ${ }^{20}$ Among postmenopausal women, the concentration of $17 \beta$ estradiol in breast tumours is at least 20 -fold higher than that in the circulation, but among premenopausal women with breast cancer, this difference was only five-fold. ${ }^{21}$ There is growing evidence that soy isoflavones may have a protective effect in terms of initiation or progression of breast cancer because they inhibit the local production of estrogens from circulating precursors in breast tissue. In vitro experiments have shown that isoflavones inhibit the activity of aromatase and $17 \beta$-hydroxysteroid dehydrogenases involved in the synthesis of estradiol from circulating androgens and estrones..$^{22,23}$ This may explain the beneficial effects of soy isoflavones on postmenopausal patients who are receiving endocrine therapy. Anastrozole is a third-generation aromatase inhibitor that has been approved for the treatment of postmenopausal breast cancer worldwide. In the study reported here, high intake of soy isoflavones reduced the risk of recurrence among patients receiving anastrozole treatment. This effect might be due to the synergistic inhibitory effects of isoflavones and anastrozole on the synthesis of estrogen.

We found that the statistically significant inverse associations between intake of soy isoflavones and breast cancer recurrence in postmenopaual women were restricted to estrogen- and progesterone-receptor positive breast cancers. This finding supports the idea of a differential role for isoflavones according to hormonal receptor status. It is consistent with the results of a large cohort study that reported a lower risk of postmenopausal estrogen- and progesterone-receptor positive breast cancer in association with higher dietary intakes of plant lignans and high exposure to enterolignans in a Western population. ${ }^{24} \mathrm{~A}$ case-control study also reported that high intakes of leafy or yellow vegetables were associated with reduced risks of postmenopausal estrogen- and progesteronereceptor positive breast cancer. ${ }^{25}$ These results were also supported by the epidemiology study of Guha and colleagues, ${ }^{19}$ who found that the risk of recurrence decreased with increasing intake of isoflavones for tumours that were estrogen- and progesterone-receptor positive, but not those that were estrogen- and progesterone-receptor negative. These findings suggest that the beneficial effects of soy isoflavones may be achieved through the estrogen and progesterone receptors.

\section{Limitations}

One limitation of this study is the possibility that other bioactive components in soy, such as soy protein, may have beneficial or harmful effects on breast tissue. Also, the results of this study may not be generalizable to other populations in which soy consumption is much lower. In addition, we did not obtain biological samples such as blood or urine for determination of soy levels, because of the difficulty of collecting such samples during a follow-up study. Such data might help to explain the influence of internal metabolism on the activity of isoflavones.

\section{Conclusions}

In this study, high dietary intake of soy isoflavones was associated with reduced risk of recurrence of disease among postmenopausal patients with estrogen- and progesterone-receptor positive breast cancer and those who were receiving anastrozole as endocrine therapy. This finding is potentially important in terms of recommendations for intake of soy isoflavones in conjunction with endocrine therapy. Large multicentre clinical trials and other observational epidemiologic studies are needed to confirm these findings.

\section{This article has been peer reviewed.}

Competing interests: Xinmei Kang and Qingyuan Zhang have received grants from the National Natural Science Foundation (China). None declared for Shuhuai Wang, Xu Huang, and Shi Jin.

Contributors: Xinmei Kang and Qingyuan Zhang were responsible for the conception and design of the study and the collection of data, as well as for writing the manuscript. Shuhuai Wang assisted with study design, recruitment of patients and data collection. Xu Huang was responsible for analyzing the data. Shi Jin assisted with verifying and interpreting the data. All of the authors participated in revising the manuscript for important intellectual content and approved the final version submitted for publication. 
Acknowledgements: The authors thank the staff of the Chinese Anticancer Association for assistance with data collection and Jie Piao for programming and statistical support.

Funding: This study was funded by grants from the National Natural Science Foundation (30901761) and the Harbin Science and Technology Bureau (2009RFQXS024).

\section{REFERENCES}

1. Tham DM, Gardner CD, Haskell WL. Clinical review 97: Potential health benefits of dietary phytoestrogens: a review of the clinical, epidemiological, and mechanistic evidence. J Clin Endocrinol Metab 1998;83:2223-35.

2. Setchell KD. Soy isoflavones - benefits and risks from nature's selective estrogen receptor modulators (SERMs). J Am Coll Nutr 2001;20 Suppl:354S-62S.

3. Atmaca A, Kleerekoper M, Bayraktar M, et al. Soy isoflavones in the management of postmenopausal osteoporosis. Menopause 2008;15:748-57.

4. Cassidy A, Hooper L. Phytoestrogens and cardiovascular disease. J Br Menopause Soc 2006; 12:49-56.

5. Ingle JN. Current status of adjuvant endocrine therapy for breast cancer. Clin Cancer Res 2001;7 Suppl:4392s-6s.

6. Colozza M, Califano R, Minenza E, et al. Aromatase inhibitors: a new reality for the adjuvant endocrine treatment of early-stage breast cancer in postmenopausa women. Mini Rev Med Chem 2008;8:564-74.

7. Ju YH, Doerge DR, Allred KF, et al. Dietary genistein negates the inhibitory effect of tamoxifen on growth of estrogen-dependent human breast cancer (MCF-7) cells implanted in athymic mice. Cancer Res 2002;62:2474-7.

8. Liu B, Edgerton S, Yang X, et al. Low-dose dietary phytoestrogen abrogates tamoxifen-associated mammary tumor prevention. Cancer Res 2005;65:879-86.

9. Constantinou AI, Lantvit D, Hawthorne M, et al. Chemopreventive effects of soy protein and purified soy isoflavones on DMBA-induced mammary tumors in female Sprague-Dawley rats. Nutr Cancer 2001;41:75-81.

10. Dai Q, Shu XO, Jin F, et al. Population-based case-control study of soyfood intake and breast cancer risk in Shanghai. Br J Cancer 2001;85:372-8.

11. Yang YX, Wang GY, Chang PX. Isoflavone content of foods. In: China food composition. Beijing (China): University Medical Publishing House; 2002. p. 331-4.

12. Hsieh FY, Lavori PW. Sample-size calculations for the Cox proportional hazard regression model with nonbinary covariates. Control Clin Trials 2000;21:552-60.

13. Hess KR. Assessing time-by-covariate interactions in proportional hazards regression models using cubic spline functions. Stat Med 1994;13:1045-62.

14. Messina M, Nagata $\mathrm{C}, \mathrm{Wu}$ AH. Estimated Asian adult soy protein and isoflavone intakes. Nutr Cancer 2006;55:1-12.

15. Shu XO, Jin F, Dai Q, et al. Soyfood intake during adolescence and subsequent risk of breast cancer among Chinese women. Cancer Epidemiol Biomarkers Prev 2001; 10:483-8.

16. Wu AH, Wan P, Hankin J, et al. Adolescent and adult soy intake and risk of breast cancer in Asian-Americans. Carcinogenesis 2002;23:1491-6.

17. Messina MJ, Wood CE. Soy isoflavones, estrogen therapy, and breast cancer risk: analysis and commentary. Nutr J 2008;7:17.

18. Boyapati SM, Shu XO, Ruan ZX, et al. Soyfood intake and breast cancer survival: a followup of the Shanghai Breast Cancer Study. Breast Cancer Res Treat 2005;92:11-7.

19. Guha N, Kwan ML, Quesenberry CP Jr, et al. Soy isoflavones and risk of cancer recurrence in a cohort of breast cancer survivors: the Life After Cancer Epidemiology study. Breast Cancer Res Treat 2009;118:395-405.

20. Nakata T, Takashima S, Shiotsu Y, et al. Role of steroid sulfatase in local formation of estrogen in post-menopausal breast cancer patients. J Steroid Biochem Mol Biol 2003;86:455-60.

21. Pasqualini JR, Chetrite GS. Recent insight on the control of enzymes involved in oestrogen formation and transformation in human breast cancer. J Steroid Biochem Mol Biol 2005;93:221-36.

22. Lacey M, Bohday J, Fonseka SM, et al. Dose-response effects of phytoestrogens on the activity and expression of $3 \beta$-hydroxysteroid dehydrogenase and aromatase in human granulosa-luteal cells. J Steroid Biochem Mol Biol 2005;96:279-86.

23. Brooks JD, Thompson LU. Mammalian lignans and genistein decrease the activities of aromatase and 17 $\beta$-hydroxysteroid dehydrogenase in MCF-7 cells. $J$ Steroid Biochem Mol Biol 2005;94:461-7.

24. Touillaud MS, Thiébaut AC, Fournier A, et al. Dietary lignan intake and postmenopausal breast cancer risk by estrogen and progesterone receptor status. J Nat Cancer Inst 2007;99:475-86.

25. Gaudet MM, Britton JA, Kabat GC, et al. Fruits, vegetables, and micronutrients in relation to breast cancer modified by menopause and hormone receptor status. Cancer Epidemiol Biomarkers Prev 2004;13:1485-94.

Correspondence to: Dr. Qingyuan Zhang, 150 Haping Rd., Harbin, China; zsci2007@hotmail.com
ATIVAN is useful for the short-term relief of manifestations of excessive anxiety in patients with anxiety neurosis. It is also useful as an adjunct for the relief of excessive anxiety that might be present prior to surgical interventions. Anxiety and tension associated with the stresses of everyday life usually do not require treatment with anxiolytic drugs.

ATIVAN is contraindicated in patients with myasthenia gravis or acute narrow angle glaucoma, and in those with known hypersensitivity to benzodiazepines.

Severe anaphylactic/anaphylactoid reactions have been reported with the use of benzodiazepines. Cases of angioedema involving the tongue, glottis or larynx have been reported in patients after taking the first or subsequent doses of benzodiazepines. Some patients taking benzodiazepines have had additional symptoms such as dyspnea, throat closing or nausea and vomiting. Some patients have required medical therapy in the emergency department. If angioedema involves the tongue, glottis or larynx, airway obstruction may occur and be fatal. Patients who develop angioedema after treatment with a benzodiazepine should not be rechallenged with the drug.

ATIVAN is not recommended for use in depressive neurosis or in psychotic reactions. Because of the lack of sufficient clinical experience, lorazepam is not recommended for use in patients less than 18 years of age. Since ATIVAN has a central nervous system depressant effect, patients should be advised against the simultaneous use of other CNS depressant drugs. Patients should also be cautioned not to take alcohol during the administration of lorazepam because of the potentiation of effects that may occur. ATIVAN should not be used during pregnancy. Since lorazepam is also a benzodiazepine derivative, its administration is rarely justified in women of childbearing potential. ATIVAN should not be administered to breast-feeding women, unless the expected benefit to the mother outweighs the potential risk to the infant.

Use of benzodiazepines, including lorazepam, may lead to potentially fatal respiratory depression.

Excessive sedation has been observed with lorazepam at standard therapeutic doses.

The most frequently reported adverse reaction to ATIVAN was drowsiness. See prescribing information for complete adverse reaction information.

The lowest effective dose of ATIVAN should be prescribed for the shortest duration possible. The risk of withdrawal and rebound phenomena is greater after abrupt discontinuation; therefore, the drug should be discontinued gradually. Withdrawal symptoms (e.g., rebound insomnia) can appear following cessation of recommended doses after as little as one week of therapy. Abrupt discontinuation of lorazepam should be avoided and a gradual, dose-tapering schedule followed after extended therapy.

ATIVAN should not be administered to individuals prone to drug abuse. Lorazepam may have abuse potential, especially in patients with a history of drug and/or alcohol abuse.

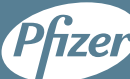

Working together for a healthier world"
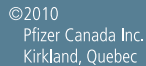

Tupfizer Inc, used under license

ATIVAN ${ }^{\circledR}$ Wyeth, owner, now part of

Pfizer Canada Inc., Licensee 